\title{
CHARACTERISTICS OF ORGANOCHLORINE POLLUTION IN THE TOPSOIL OF THE DAWEN RIVER WATERSHED AND POTENTIAL RISK ASSESSMENT IN CHINA
}

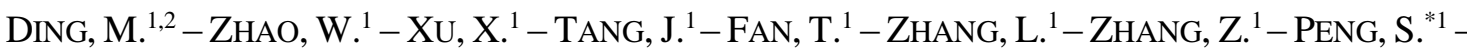 \\ XU, L. ${ }^{1}$ \\ ${ }^{1}$ Tourism College, Taishan University, Tai'an 271000, China \\ ${ }^{2}$ Laboratory for Marine Geology, Qingdao National Laboratory for Marine Science and \\ Technology, Qingdao 266000, China \\ *Corresponding author \\ e-mail: shuzhenpeng@sohu.com
}

(Received 22 $2^{\text {nd }}$ Jul 2019; accepted $15^{\text {th }}$ Nov 2019)

\begin{abstract}
We detected presence of 23 types of organochlorine pesticides (OCPs) in 21 mixed samples obtained along the banks of Dawen River in China by gas chromatography. we analyzed the spatial distribution of OC residues, potential source, and the ecological and health risks. The results show that OCPs were detected in all the 21 sampling sites, and $86.6 \%$ of the 23 types of OCPs had a detection rate above $90 \%$. Furthermore, the analysis of the single-factor pollution index shows that $14 \%$ of the sampling sites were mildly polluted, whereas $4.8 \%$ had medium-level pollution. Analysis of the Nemerow's multifactor index shows that $9.5 \%$ of the sampling sites were mildly polluted. We found that Dawenkou Town and Wenyang Town are severely polluted, whereas Ciyao Town and Jiangji Town are less polluted by arcgis12.0 spatial analysis. Compared to other regions in China, the HCHs content is at a low level but DDTs at a high level in the study area. Assessment of health risks of OCP residues shows that the carcinogenic risk and the non-carcinogenic risk caused by exposure to OCPs is negligible. OCPs are primarily obtained from previous residues and recent use of lindane.
\end{abstract}

Keywords: OCPs residues, DDT, HCHs, health risks

\section{Introduction}

Chlorine in organic compounds is referred to as organochlorine. Organochlorine pesticides (OCPs), a type of persistent organic pollutants, have been drawn considerable research attention because of the pollution and environmental harm they have caused. Once OCPs enter the soil, the soil is contaminated, and because OCPs are hard to decompose and accumulate, they will enter the human body through the food chain and cause intoxication. OCPs are harmful to both the ecological system and human health, resulting in nervous system disorders, cancer, reproductive abnormalities, and congenital defects (Chen et al., 2017), Although the Chinese government banned OCPs in the 1980s, studies in recent years have revealed that HCHs and DDTs were still found in the soil and water in many regions across China (An et al., 2005; Cheng, 2014; Cui et al., 2014; Cheng et al., 2018; Cui et al., 2012, 2014; Chen et al., 2011; Dai et al., 2012).

Dawen River watershed, which is famous for high and stable crop yields, has been included in the ecological restoration program of the Mount Tai area in Shandong province of China. As an important part of the urban environment, the suburban soil has significant influence over the quality of the environment and ecological functions of a city. Note that OCPs residues in soil will affect the safety of crops and may cause food scarcity in cities and threaten human health (Cui et al., 2014). 
Using the topsoil in Wenyang Field as the research object, we performed pollution analysis, health risk analysis, and ecological risk analysis to identify the pollution condition and potential risks in Wenyang Field. We believe this study will provide effective solutions for soil restoration and provide a scientific basis for sustainable soil utilization and the protection of human health.

\section{Materials and methods}

\section{Study area description}

Wenyang Field (Fig. 1), the fertile land along the banks of Dawen River in its middle and lower reaches in china, was selected as the research area. Wenyang Field has a flat terrain and covers an area of $14 \mathrm{~km}^{2}$. Feicheng City, Ningyang County, and Daiyue District located in Wenyang Field. It is subject to the warm temperate continental monsoon climate with high temperature coinciding with high precipitation, which facilitates the growth of crops. The major soil types of soil in this area include cinnamon soil and brown soil. The soil layer is thick and the soil is fertile. Dawen River, the largest branch of Yellow River in its lower reach, flows through Wenyang Field, while the underground water level is three to four meters deep. These natural conditions provide Wenyang Field with abundant water and fertile soil. As an important crop-yielding area in Taiwan, Wenyang Field is known as the "bread basket of Mount Tai" and supplies over 10,000 tons of corns and wheat to China; it is also rich in mineral resources such as gypsum and halite.

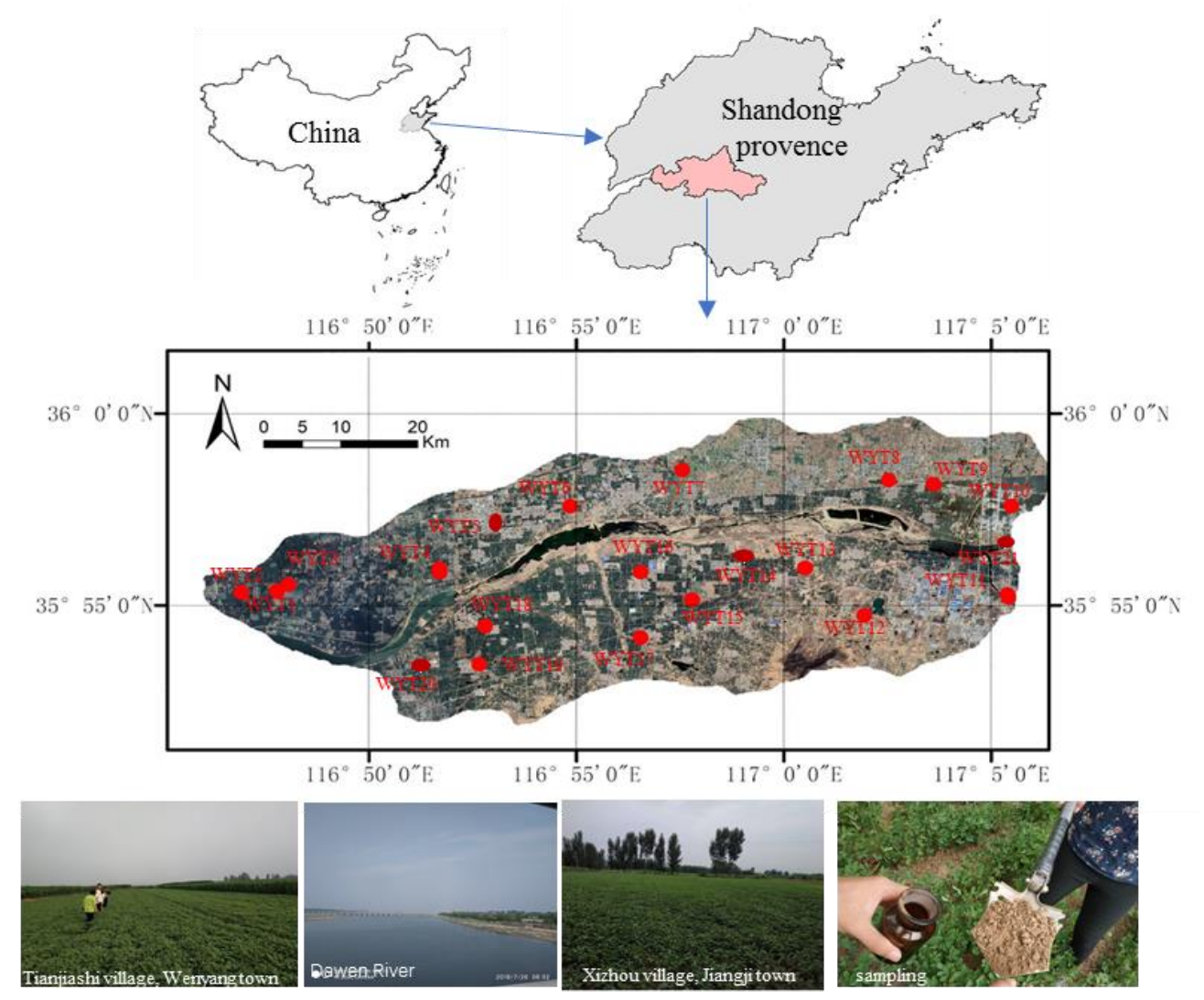

Figure 1. Location of study area, distribution of sampling site sand sampling photos in Dawen River Basin in China 


\section{Sample collection}

The sampling points are located at 21 locations on both sides of the Dawen River (Table 1, Fig. 1). Preliminary surveys of the Dawen River watershed were conducted using GPS, and 21 mixed soil samples were collected according to "Technical Guidelines for Environmental Site Investigation" (HJ25.1-2014) in July 2018. Fivepoint sampling method was used to collect the samples, 5 samples are collected at each location to be mixed into one sample. and the sampling depth was from 0 to $20 \mathrm{~cm}$. Details of specific sampling point locations and vegetation. The $200 \mathrm{~g}$ soil was sealed in brown wild-mouth bottles $(250 \mathrm{ml})$ after being collected and stored in lab freezers at $4{ }^{\circ} \mathrm{C}$. To test the physical and chemical indicators of the soil, a soil sample of $1 \mathrm{~kg}$ was collected and packed in the sampling bag. The collected soil samples were transported to the Lunan Institute of Geo-engineering Investigation of Shandong for additional tests.

In this paper, SPSS 12.0 software is used for data statistical analysis, and arcgis12.0 Kriging difference method is used for spatial analysis (Ma et al. 2015).

Table 1. Sampling sites, number of the samples and land use of soil sampling in Dawen River Basin in China

\begin{tabular}{|c|c|c|c|c|c|}
\hline Samples & No. & Sampling point (n) & Longitude & Latitude & Lan \\
\hline WYT1 & 5 & Sihechakou village, Wenyang town & $116.79648^{\circ} \mathrm{E}$ & $35.92304^{\circ} \mathrm{N}$ & Corn field \\
\hline WYT2 & 5 & Sanjiakou new village, Anjiazhuang town & $116.78217^{\circ} \mathrm{E}$ & $35.92241^{\circ} \mathrm{N}$ & Soybean field \\
\hline WYT3 & 5 & Donggaoyu village, Wenyang town & $116.80101^{\circ} \mathrm{E}$ & $35.92575^{\circ} \mathrm{N}$ & sorghum field \\
\hline WYT4 & 5 & Maidongshi village, Wenyang town & 116.8 & $35.93099^{\circ} \mathrm{N}$ & Vegetable plot \\
\hline WYT5 & 5 & Tianjiashi village, Wenyang town & $116.86170^{\circ} \mathrm{E}$ & $35.93279^{\circ} \mathrm{N}$ & Peanut field \\
\hline WYT6 & 5 & Beidian village, Wenyang town & $116.91425^{\circ} \mathrm{E}$ & $35.95981^{\circ} \mathrm{N}$ & Okra field \\
\hline WYT7 & 5 & Xiama village, Mazhuang town & $116.95935^{\circ} \mathrm{E}$ & $35.97548^{\circ} \mathrm{N}$ & Soybean field \\
\hline WYT8 & 5 & West of Yuejiazhuang, Dawenkou town & $117 .($ & $17^{\circ} \mathrm{N}$ & Corn field \\
\hline WYT9 & 5 & East Yuejia, Dawenkou town & $117.06027^{\circ} \mathrm{E}$ & $35.96941^{\circ} \mathrm{N}$ & Celery field \\
\hline WYT10 & 5 & Xinghua village, Wenkou town & $117.09148^{\circ} \mathrm{E}$ & $35.95994^{\circ} \mathrm{N}$ & Corn field \\
\hline WYT11 & 5 & Qijiazhuang, Ciyao town & $117.08991^{\circ} \mathrm{E}$ & $35.92143^{\circ} \mathrm{N}$ & Peanut field \\
\hline WYT12 & 5 & Lujiahuaguan village, Ciyao town & $117.03247^{\circ} \mathrm{E}$ & $35.91231^{\circ} \mathrm{N}$ & Peanut field \\
\hline WYT13 & 5 & Xizhou village, Jiangji town & $117.00872^{\circ} \mathrm{E}$ & $35.93303^{\circ} \mathrm{N}$ & Peanut field \\
\hline WYT14 & 5 & Dahuzhuang village, Jiangji town & $117.00859^{\circ} \mathrm{E}$ & $35.93293^{\circ} \mathrm{N}$ & Ginger field \\
\hline WYT15 & 5 & Hengjiazhuang, Jiangji town & 116.9 & $35.91915^{\circ} \mathrm{N}$ & Corn field \\
\hline WYT16 & 5 & Tianfu Village, Jiangji town & $116.94264^{\circ} \mathrm{E}$ & $35.93113^{\circ} \mathrm{N}$ & Corn field \\
\hline WTY17 & 5 & Zhangjiadian, Gucheng & $116.94264^{\circ} \mathrm{E}$ & $35.90261^{\circ} \mathrm{N}$ & Corn field \\
\hline WYT18 & 5 & Chenjiadian, Gucheng & $116.880110^{\circ} \mathrm{E}$ & $35.90768^{\circ} \mathrm{N}$ & Peanut field \\
\hline WYT19 & 5 & Xingquan village, Gucheng town & $116.87773^{\circ} \mathrm{E}$ & $35.89111^{\circ} \mathrm{N}$ & Corn field \\
\hline WYT20 & 5 & Panxinzhuang, Gucheng town & $116.83494^{\circ} \mathrm{E}$ & $35.87919^{\circ} \mathrm{N}$ & Corn field \\
\hline WYT21 & 5 & Zhengjia village, Ciyao town & $117.09045^{\circ} \mathrm{E}$ & $35.82018^{\circ} \mathrm{N}$ & Wetland \\
\hline
\end{tabular}

\section{Methods}

The samples were tested at the Nunan Institute of Geo-engineering Investigation of Shandong and 23 types of OC compounds were analyzed. The GC-ECD method was used to detect OCPs in the soil. The detection and testing tools included the Agilent 7890B-5977B mass spectrometer, the Thermo ASE 350 pressurized fluid extractor, the SUPELCO VISIPREP $24^{\mathrm{TM}}$ DL solid-phase extraction device, and the HP-5 
chromatographic column. The reagents used included 20 types of standard mixtures of OC solvents, and the testing procedure was as follows. The samples were dried and grinded before being screened through a $250-\mu \mathrm{m}$ sieve (No. 60 sieve). The sample was mixed with diatomaceous earth $(1 \mathrm{~g})$ and the mixture was added to the extraction cell (22 ml) before pressurized fluid extraction was conducted according to the HJ783-2016 standards. After extraction, n-hexane was used to wash the tube wall of the concentrator exposed during the nitrogen blowing process to concentrate $\mathrm{n}$-hexane to $1 \mathrm{ml}$. The eluent was then transferred to the column and $2 \mathrm{ml}$ of $\mathrm{n}$-hexane was used to wash the concentrator several times, and then all the eluent was transferred into the column (if desulfurization was required, the solvent would be sunk in copper sheets for $\sim 5 \mathrm{~min}$ ). The eluents were collected and concentrated before the internal standard was added for testing. When drawing the curves, five sample bottles of $1.5 \mathrm{ml}$ were used to prepare solvents of five different concentrations by mixing the OCPs standard intermediate solution, the substitute intermediate solution, internal standard intermediate solution, and n-hexane solution extracted by the pipette. The mass concentrations of OCPs and substitutes for the five solvents were 20,50,100, 200 and $500 \mathrm{ng} / \mathrm{ml}$, and the internal standard mass concentration was $20.0 \mathrm{ng} / \mathrm{ml}$.

\section{Results}

\section{Characteristics of OCPs residues}

Table 2 shows the conditions of OCPs residues in the soil of Wenyang Field in china. The total content of OCPs residues ( $\Sigma$ OCPs) is between $n d$ and $197.01 \mathrm{ng} / \mathrm{g}$. The detection rate of OCPs in the 21 sampling sites in Wenyang Field reached 100\%, and 23 types of OCPs were detected. The most prevalent OCPs detected in the soil of Wenyang Field measured by the average content are DDTs $(53.84 \mathrm{ng} / \mathrm{g})$, endrin aldehyde $(18.47 \mathrm{ng} / \mathrm{g})$, and heptachlor epoxide $(17.07 \mathrm{ng} / \mathrm{g})$.

\section{$\mathrm{HCHs}$}

The detection rate of $\mathrm{HCHs}$ (such as $\alpha-, \beta-, \gamma-$, and $\delta-\mathrm{HCH}$ ) is $100 \%$ with the residue content between 1.58 and $15.95 \mathrm{ng} / \mathrm{g}$ (average of $4.23 \mathrm{ng} / \mathrm{g}$ ). The largest content of HCHs was observed at WYT10 (near the glass manufacturing plant), while the content of $\mathrm{HCHs}$ in the topsoil of Wenyang Field was far below $100 \mathrm{ng} / \mathrm{g}$, which is the threshold value for agricultural land pollution as stipulated in "Soil Environment Quality Standard" (GB15618-2018). The content of HCHs in the topsoil of Wenyang Field is below that at Jining in Shandong (5.73 ng/g) (Pang et al., 2009), Henan province (12.55 ng/g) (Cheng et al., 2018), Baotou (8.37 ng/g) (Meng et al., 2017a), Inner Mongolia (8.63 ng/g) (Meng et al., 2017b), Changchun (10.95 ng/g) (Xiao, 2017), and Yangtze River Delta (7.73 ng/g) (Shi et al., 2016); however, the content of HCHs is above that in Shanghai $(2.19 \mathrm{ng} / \mathrm{g}$ ) (Cheng et al., 2018). This comparison indicates that the HCHs pollution in the soil of Wenyang Field is still at a low level. The HCH isomers have different physical, chemical, and toxic properties; furthermore, in terms of the dichlorination and degradation rate, $\gamma-\mathrm{HCH}>\alpha-\mathrm{HCH}>\delta-\mathrm{HCH}>\beta-\mathrm{HCH}$. However, the results of this study show that the detection rate of $\beta-\mathrm{HCH}$ is the highest, and $\beta$ $\mathrm{HCH}>\gamma-\mathrm{HCH}>\alpha-\mathrm{HCH}>\delta-\mathrm{HCH}$ in terms of the average residue of HCHs. According to Figure $2 a$, which shows the distribution of $\mathrm{HCH}$ content, the $\mathrm{HCH}$ content is high in Wenkou Town and Ciyao Town but relatively low in the western area. 
Table 2. Summary of OCPs concentrations in topsoil in Dawen River Basin in China $(n=21)$ (units for soil samples are $\left.n g g^{-1} d w\right)$

\begin{tabular}{c|c|c|c|c|c|c}
\hline Compounds & Mean & $\begin{array}{c}\text { Standard } \\
\text { deviation }\end{array}$ & Minimum & Maximum & $\begin{array}{c}\text { Coefficient } \\
\text { of variation }\end{array}$ & $\begin{array}{c}\text { Detection } \\
\text { rate }\end{array}$ \\
\hline HCB & 0.06 & 0.03 & 0.03 & 0.16 & 0.52 & $100 \%$ \\
$\alpha$-HCH & 0.76 & 0.78 & 0.12 & 3.75 & 1.02 & $100 \%$ \\
$\beta$-HCH & 2.18 & 2.25 & 0.75 & 8.76 & 1.03 & $100 \%$ \\
$\gamma$-HCH & 0.98 & 0.87 & 0.25 & 3.40 & 0.89 & $100 \%$ \\
$\delta$-HCH & 0.31 & 0.33 & 0.10 & 1.43 & 1.07 & $100 \%$ \\
HCHs & 4.23 & 3.95 & 1.58 & 15.95 & 0.93 & $100 \%$ \\
Heptachlor & 1.89 & 1.17 & 0.75 & 5.83 & 0.62 & $100 \%$ \\
Aldrin & 0.07 & 0.05 & 0.00 & 0.26 & 0.77 & $95.24 \%$ \\
Dicofol & 3.69 & 3.74 & 0.40 & 15.79 & 1.01 & $100 \%$ \\
Epoxyheptachloride & 17.07 & 4187.00 & 0.00 & 194.39 & 2.45 & $90.48 \%$ \\
г- chlordane & 0.02 & 0.02 & 0.00 & 0.09 & 0.66 & $95.24 \%$ \\
a- chlordane & 0.02 & 0.01 & 0.00 & 0.05 & 0.52 & $90.48 \%$ \\
dieldrin & 1.93 & 3.35 & 0.59 & 16.45 & 1.74 & $100 \%$ \\
Isodrin & 0.78 & 1.44 & 0.00 & 5.58 & 1.84 & $38.10 \%$ \\
Endosulfan 2 & 0.56 & 1.08 & 0.00 & 4.30 & 1.19 & $95.27 \%$ \\
p,p'-DDD & 2.04 & 3.49 & 0.26 & 15.58 & 1.71 & $100 \%$ \\
o,p'-DDT & 0.44 & 0.40 & 0.08 & 1.71 & 0.90 & $100 \%$ \\
p,p'-DDT & 47.53 & 48.21 & 5.83 & 192.65 & 1.01 & $100 \%$ \\
DDTs & 53.84 & 50.08 & 8.40 & 197.01 & 0.93 & $100 \%$ \\
Endrin aldehyde & 18.47 & 12.01 & 3.85 & 52.98 & 0.65 & $100 \%$ \\
Endosulfan sulphate & 0.91 & 3.27 & 0.00 & 15.13 & 3.59 & $61.90 \%$ \\
Isodiazone & 0.25 & 0.94 & 0.00 & 4.29 & 3.75 & $76.19 \%$ \\
methoxychlor & 1.18 & 1.08 & 0.08 & 3.33 & 0.92 & $100 \%$ \\
Mirex & 0.44 & 0.70 & 0.00 & 3.39 & 1.60 & $100 \%$ \\
\hline
\end{tabular}
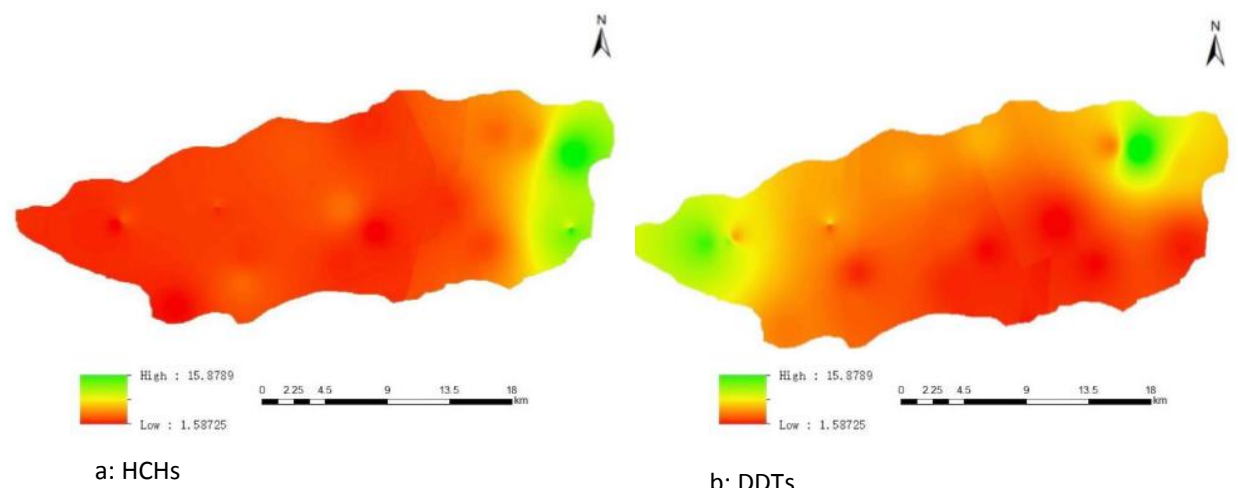

b: DDTs

Figure 2. Distribution of HCHs and DDTs content in Dawen River Basin in China

\section{DDTs}

The detection rate of DDTs, including DD, DDE, and DDT, is 100\% in the study area, and the residue content is between 8.4 and $197.01 \mathrm{ng} / \mathrm{g}$ (average of $53.84 \mathrm{ng} / \mathrm{g}$ ). 
The highest content occurs at WYT9 (a celery-growing field). Note that the residue content of DDTs is higher than that of HCHs, and HCHs and DDTs have the same variable coefficient of 0.93 . The average content of DDTs in the topsoil of Wenyang Field is $51 \%$ of the average content of all organic pollutants. The DDTs content in the topsoil of some sampling sites is already $>100 \mathrm{ng} / \mathrm{g}$, which is the threshold value for agricultural land pollution risks stipulated in "Soil Environment Quality Standard" (GB15618-2018). Note that the content of DDTs in the topsoil of Wenyang Field is higher than that at Jining (17 ng/g) (Pang et al., 2009), Shanghai (13.15 ng/g) (Jiang et al., 2010), Henan (8.97 ng/g) (Cheng et al., 2018), Baotou (9.51 ng/g) (Meng et al., 2017a), Inner Mongolia (9.71 ng/g) (Meng et al., 2017b) and Yangtze River Delta (Shi Lei et al., 2016); however, it is below that at Yantai (160 ng/g) (Dai et al., 2012) and Songhuajiang in Jilin (92 ng/g) (Liu et al., 2013). The comparison indicates that the DDT pollution in the soil of Wenyang Field is at a high level. The four derivatives of DDTs by average content are ranked as follows: p, $\mathrm{p}^{\prime}-\mathrm{DDT}>\mathrm{p}, \mathrm{p}^{\prime}$-DDE $>\mathrm{p}, \mathrm{p}^{\prime}-$ DDD $>0, \mathrm{p}^{\prime}$-DDT, and the content of p, $\mathrm{p}^{\prime}$-DDT is the highest. Figure $2 b$ shows that the content of DDTs is high in Anjiazhuang Town and Dawenkou Town, but relatively low in Jiangji Town and Ciyao Town.

\section{Discussion}

\section{OCPs pollution analysis}

Single-factor pollution index analysis

The single-factor index method (Chen et al., 2011; Wu, 2012) and the equation $P_{i}=C_{i} / S_{i}$ were used to calculate the single-factor pollution index $P_{i}$ of the pollutant $i$ :

$$
p_{i}=\frac{c_{i}}{s_{i}}
$$

Figure 3 shows the calculation results, and the single-factor pollution index of $\mathrm{HCHs}$ is generally seen to be lesser than that of DDTs. The curve for DDTs in Figure 3 shows sharp fluctuation, which indicates the pollution level of DDTs varies considerably from one region to another. While $14 \%$ of sampling sites showed mild pollution, Xiaohou Village showed the highest single-factor pollution index of $\sim 1.9$; hence, it is considered under medium-level pollution. Figure 4 shows that the level of pollution is high in Dawen Town and Wenyang Town, but relatively low in Ciyao Town and Jiangji Town.

\section{Nemerow's pollution index analysis}

According to the Nemerow's pollution index method, we used the equation to calculate the composite soil pollution index $P_{N}$ :

$$
P_{N}=\sqrt{\frac{P_{i}^{2}+P_{i(\max )}^{2}}{2}}
$$

Figure 5 shows the calculation results in which $9.5 \%$ of sampling sites are mildly polluted with the highest index in Xiaohou Village. As shown in Figure 6, the pollution level in Dawenkou Town and Wenyang Town is high, but it is relatively low in Ciyao Town and Jiangji Town. 


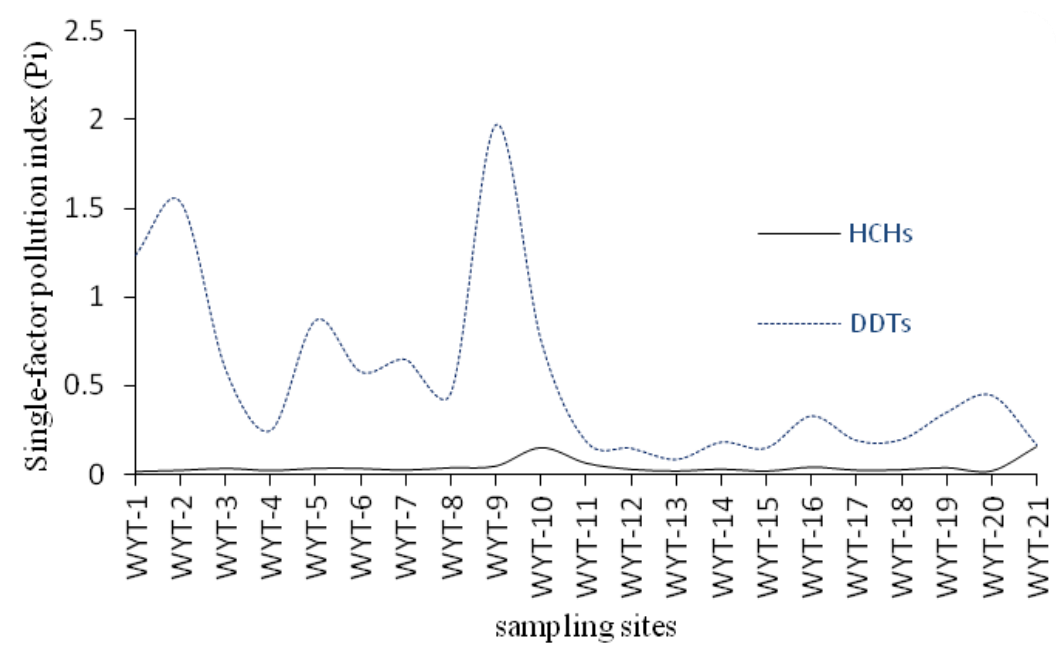

Figure 3. Single-factor indices of OCPs in the topsoil of Wenyang Field in China

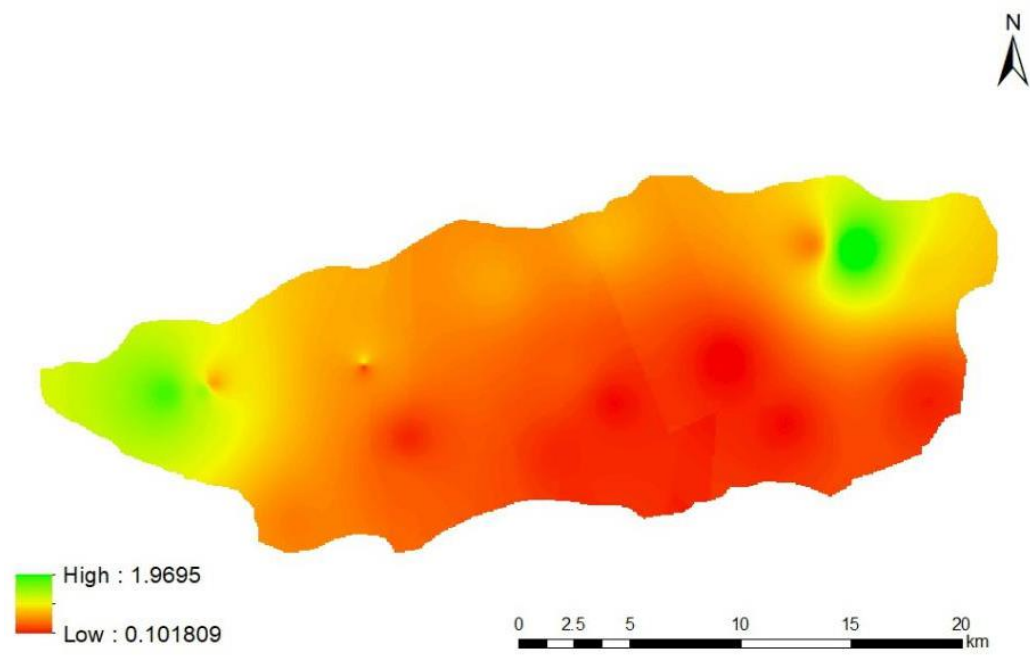

Figure 4. Spatial interpolation of DDT single-factor pollution in Dawen River Basin in China

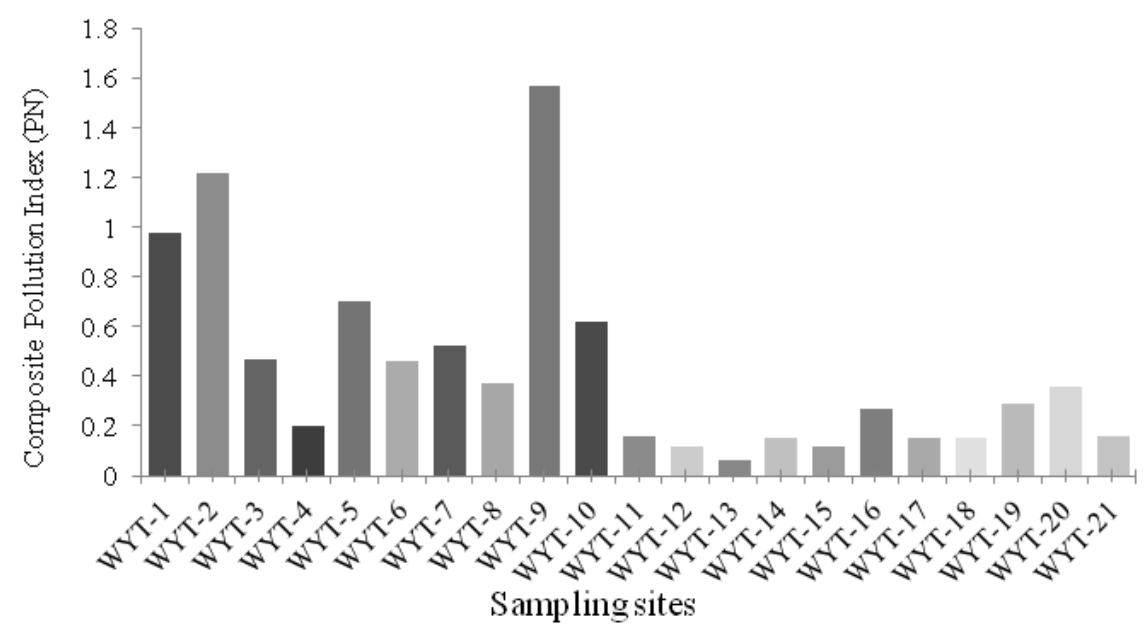

Figure 5. Composite OCPs pollution index in the topsoil of Wenyang Field in China 

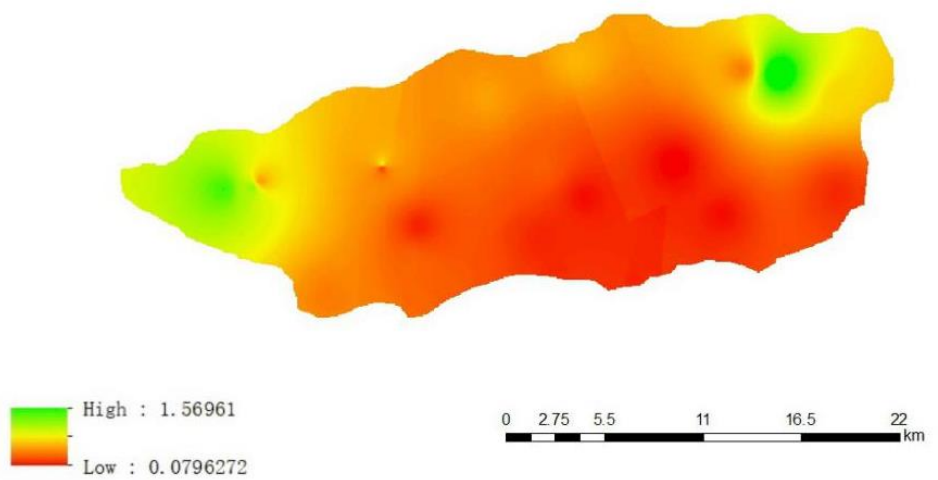

Figure 6. Interpolation of Nemerow's pollution index in Dawen River Basin in China

\section{Assessment of ecological risks of OCPs}

We assessed the ecological risks of OCPs according to the concept of adverse biological effects proposed (Long et al., 1995). Effect range low (ERL) and effect range median (ERM), proposed in the study by Long et al., are used as threshold values to identify the level of ecological risks of pollutants. If the value of a pollutant is lesser than ERL, it has no obvious biological toxicity and the ecological risk is < 10\%; if the value falls between ERL and ERM, the pollutant has potential toxic effects; and if the value is higher than ERM, it is biologically toxic and its ecological risk is $>75 \%$ (Zhu et al., 2012; Lin et al., 2011).

The data obtained in tests in this study are compared with ERL and ERM. Note that in 20 of the 21 samples, i.e., $95.24 \%$ of samples, the value of p,p'-DDT is higher than ERM, which indicates that it has a very high level of ecological risk. Among the 21 samples, the value of p,p'-DDE is lower than ERL in nine samples; however, the value of p,p'-DDE in the other 12 is between ERL and ERM, i.e., $42.86 \%$ and $57.14 \%$ of samples, respectively, which indicates that p,p'-DDE has a relatively high level of ecological risk. Note that in 16 of the 21 samples, i.e., $76.19 \%$ of samples, the value of p,p'-DDD is lower than ERL. Thus, the ecological risks posed by OCPs in the soil of Wenyang Field needs to be examined.

Assessment of ecological risks of DDTs is based on studies (Jongbloed et al., 1996). By designing a simplified food web, Jongbloed et al. identified the threshold content of DDTs in soil, which would cause secondary poisoning: $10 \mu \mathrm{g} / \mathrm{kg}$ for soil worms, $11 \mu \mathrm{g} / \mathrm{kg}$ for birds, and $190 \mu \mathrm{g} / \mathrm{kg}$ for mammals (Jongbloed et al., 1996). In most of the sampling sites, the content of DDT in soil is higher than the maximum allowable content of DDTs residues for soil worms and birds; therefore, the ecological risk they pose to soil worms and birds is high. However, the content of DDTs in most of the sampling sites is below the maximum allowable content of DDTs residues for mammals; therefore, the ecological risk DDTs pose to mammals is relatively low.

\section{Assessment of health risks of OCPs}

Human health risk research is an important content of organic pollution research, and the risk can be used as an important basis for the next soil use. Most of them use the 
health risk model recommended by the EPA of the United States for evaluation, and the results show that the health risk in most regions is relatively small (Liu et al., 2017; Zhang, 2016; Cheng, 2014).

Based on the health risk assessment model proposed by United States Environmental Protection Agency (USEPA), this study assesses the human health risks of OCPs residues in soil. The human body can be exposed to OCPs in soil via three channels: ingestion through the mouth, dermal contact, and inhaling of dust or volatile gases. The parameters for calculating the average daily dosage are determined from the guiding documents issued by USEPA (USEPA,1997, 2002, 2010) and the studies by Niu, et al. (Niu et al., 2013). The equations to calculate the average daily dosage (ADD, $\mathrm{mg} / \mathrm{kg}^{-1}$ day $^{-1}$ ) of OCPs that the human body is exposed to by the three pathways are listed as Equations 3-5 (USEPA, 1996):

$$
\begin{aligned}
& \mathrm{ADD}_{\text {ingest }}=\frac{\mathrm{C}_{\text {soil }} \times \text { IngRXEFXED }}{\text { PEFXBWXAT }} \times C F \\
& \mathrm{ADD}_{\text {dermal }}=\frac{\mathrm{C}_{\text {soil }} \times \text { SAXAFXABSXEFXED }}{\text { BW } \times A T} \times C F \\
& A D D_{\text {inhale }}=\frac{\mathrm{C}_{\text {soil }} \times \text { InhRXEFXED }}{\text { PEFXBWXAT }}
\end{aligned}
$$

Using the hazard index (HI) method, this study evaluates the non-carcinogenic risk of OCPs; moreover, by dividing the reference daily dosage from each exposure channel $\left(\mathrm{ADD}_{\text {ing }}, \mathrm{ADD}_{\text {dermal}}\right.$, and $\left.\mathrm{ADD}_{\text {inh }}\right)$ by the actual daily dosage from each channel, this study calculates the hazard quotient (HQ) of each channel of exposure (Zhang Jingjing.2016). By multiplying the life average daily dosage (LADD) and the cancer slope factor (SF), this study calculates the carcinogenic risk (Risk). The calculation equations are as follows (Eqs. 6-9) (USEPA, 2002):

$$
\begin{gathered}
H Q=\frac{\mathrm{ADD}}{\mathrm{RFD}} \\
\mathrm{HI}=\sum H Q_{i} \\
\text { Risk }_{i}=L A D D \times S F_{i} \\
\text { Risk }=\sum \text { Risk }_{i}
\end{gathered}
$$

$H I$ means the overall non-carcinogenic risk of exposure to OCPs via all three channels. When $H I$ or $H Q$ is $<1$, the risk is considered low or negligible; however, when $H I$ or $H Q$ is $>1$, it is considered that there are non-carcinogenic risks. Risk refers to the level of carcinogenic risk, i.e., the probability of developing cancer. $S F$ refers to the cancer slope factor, which indicates the maximum possibility of developing cancer associated with exposure to a certain dosage of a pollutant. When Risk is $<10^{-6}$, the risk of cancer is considered very low; when Risk is between $10^{-6}$ and $10^{-4}$, the risk of cancer is considered low; and when Risk is $>10^{-1}$, the risk of cancer is considered high (Niu et al., 2013). The reference dosage and carcinogenic slope factor for each channel are obtained from the Supplemental Guidance for Developing Soil Screening Levels for Superfund Sites issued by USEPA in 2010 (USEPA, 2010). 
According to the calculation results, both among children and adults, the three exposure channels are ranked as follows by the dosage of OCPs that enter the human body: ingestion through the month $>$ the dosage from inhaling $>$ the dosage from dermal contact. Ingestion is the major channel of exposure to OCPs. By ranking the detected daily dosage of OCPs, the dosage in children from all these three channels is higher than that in adults. In terms of the dosage of exposure, leading to non-carcinogenic risks, the different types of OCPs are ranked as follows: DDT, HCHs, $p, p^{\prime}-\mathrm{DDE}, \beta-$ $\mathrm{HCH}, \mathrm{p}, \mathrm{p}^{\prime}-\mathrm{DDD}, \gamma-\mathrm{HCH}, \alpha-\mathrm{HCH}$. Table 3 shows the average daily dosages of these OCPs.

Table 3. Average daily exposure of three pathways $\left(\mathrm{mg} \mathrm{kg}^{-1}\right.$ day $\left.{ }^{-1}\right)$

\begin{tabular}{c|c|c|c|c|c|c}
\hline & ADD $_{\text {ing }}$ & ADD $_{\text {dermal }}$ & ADD $_{\text {inh }}$ & ADD $_{\text {ing }}$ & ADD $_{\text {dermal }}$ & ADD $_{\text {inh }}$ \\
\hline DDTs & $5.26 \mathrm{E}-08$ & $1.47 \mathrm{E}-08$ & $2.32 \mathrm{E}-08$ & $2.25 \mathrm{E}-08$ & $8.99 \mathrm{E}-09$ & $2.19 \mathrm{E}-08$ \\
$\mathrm{HCHs}$ & $4.64 \mathrm{E}-09$ & $1.30 \mathrm{E}-09$ & $2.04 \mathrm{E}-09$ & $1.99 \mathrm{E}-09$ & $7.93 \mathrm{E}-10$ & $1.94 \mathrm{E}-09$ \\
$\mathrm{p}, \mathrm{p}^{\prime}-\mathrm{DDE}$ & $4.21 \mathrm{E}-09$ & $1.18 \mathrm{E}-09$ & $1.86 \mathrm{E}-09$ & $1.80 \mathrm{E}-09$ & $7.19 \mathrm{E}-10$ & $1.76 \mathrm{E}-09$ \\
$\beta$-HCH & $2.39 \mathrm{E}-09$ & $6.69 \mathrm{E}-10$ & $1.05 \mathrm{E}-09$ & $1.02 \mathrm{E}-09$ & $4.08 \mathrm{E}-10$ & $9.97 \mathrm{E}-10$ \\
$\mathrm{p}, \mathrm{p}$ '-DDD & $2.23 \mathrm{E}-09$ & $6.25 \mathrm{E}-10$ & $9.85 \mathrm{E}-10$ & $9.57 \mathrm{E}-10$ & $3.82 \mathrm{E}-10$ & $9.33 \mathrm{E}-10$ \\
$\gamma$-HCH & $1.08 \mathrm{E}-09$ & $3.02 \mathrm{E}-10$ & $4.76 \mathrm{E}-10$ & $4.62 \mathrm{E}-10$ & $1.84 \mathrm{E}-10$ & $4.50 \mathrm{E}-10$ \\
$\alpha-\mathrm{HCH}$ & $8.36 \mathrm{E}-10$ & $2.34 \mathrm{E}-10$ & $3.69 \mathrm{E}-10$ & $3.58 \mathrm{E}-10$ & $1.43 \mathrm{E}-10$ & $3.49 \mathrm{E}-10$ \\
\hline
\end{tabular}

According to equations and relevant parameters of the three channels, the different types of OCPs are ranked as DDTs $>\mathrm{HCHs}>\alpha-\mathrm{HCH}>\beta-\mathrm{HCH}>\mathrm{p}, \mathrm{p}^{\prime}-\mathrm{DDE}>\mathrm{p}, \mathrm{p}^{\prime}-$ $\mathrm{DDD}>\gamma-\mathrm{HCH}$ in terms of the carcinogenic risk they incur. The carcinogenic risk associated with exposure to OCPs through all the three channels among children is higher than that among adults. As shown by the calculation result, the value of carcinogenic risks associated with exposure to OCPs through different channels is far below $10^{-6}$, which indicates that the carcinogenic risk of OCPs in the topsoil of Wenyang Field is small and negligible; hence, it will not pose a threat to human health.

According to certain parameters and calculation of the equations, both among children and adults, the value of non-carcinogenic risk associated with exposure to OCPs via the ingestion channel is higher than that through the dermal contact channel. Note that the value of non-carcinogenic risk associated with exposure to OCPs through the inhaling channel has not been obtained because of the lack of corresponding parameters. In terms of the non-carcinogenic risk associated with exposure through the channels of ingestion and dermal contact, the different types of OCPs are ranked as DDTs $>\mathrm{HCHs}>\beta-\mathrm{HCH}>\gamma-\mathrm{HCH}>\alpha-\mathrm{HCH}$. The non-carcinogenic risk (represented by HI) of each type of OCPs is far below 1, which indicates that the non-carcinogenic risk posed by OCPs in the topsoil of Wenyang Field is small and almost negligible.

\section{Pollution source analysis}

DDTs have two biodegradation mechanisms in soil, i.e. either DDT is degraded to DDD through reductive dichlorination in anaerobic conditions or it is degraded to DDE through dehydrochlorination in aerobiotic conditions. Note that DDD and DDE are less toxic than DDT, and DDE is very stable. Generally, in the soil contaminated by DDTs, the ratio of (DDE + DDD)/DDT will exceed 1, which indicates that the DDTs have not been used for a long time and that there is no new source of DDT pollution (Cui et al., 
2014). Thus, the ratio of (DDE + DDD)/DDT can be used to identify whether DDTs are used in the study area. In this study, the ratio (Table 4) of the 21 sampling sites is below 1(0-0.6), which means DDTs are used recently. If the ratio of (p,p'-DDT + o,p'DDT)/DDTs is below 0.5, it indicates that the DDT pesticides are used long ago and there is no new source of pollution. The calculated ratio (Table 4) in the 21 sampling sites is above 0.5(0.6-1.0), which means that it has not been long since usage of DDTs in most areas of the Dawen River watershed, and DDTs are recently used in the 21 sampling sites (Cui et al., 2014). These two ratios lead to the same conclusion that it has not been long since DDTs are used in the topsoil of the Dawen River watershed.

Table 4. Proportions of DDTs for source identification in surface soil of the study area $\left(\right.$ ng $\left.g^{-1}\right)$

\begin{tabular}{c|c|c|c|c|c|c|c}
\hline Samples & p,p'-DDE & p,p'-DDD & p,p'-DDT & o,p'-DDT & DDTs & $\begin{array}{c}\text { (p,p'-DDD+p,p'- } \\
\text { DDE)/DDTs }\end{array}$ & $\begin{array}{c}\text { (p,p'-DDT+o,p'- } \\
\text { DDT)/DDTs }\end{array}$ \\
\hline WYT-1 & 9.9 & 0.9 & 112.3 & 0.4 & 123.5 & 0.1 & 0.9 \\
WYT-2 & 7.4 & 1.3 & 143.8 & 0.9 & 153.4 & 0.1 & 0.9 \\
WYT-3 & 3.7 & 0.5 & 53.8 & 0.9 & 58.9 & 0.1 & 0.9 \\
WYT-4 & 0.6 & 0.3 & 23.6 & 0.3 & 24.8 & 0.0 & 1.0 \\
WYT-5 & 9.5 & 2.0 & 75.0 & 0.4 & 86.9 & 0.2 & 0.9 \\
WYT-6 & 9.1 & 2.7 & 45.1 & 0.8 & 57.8 & 0.3 & 0.8 \\
WYT-7 & 3.0 & 0.8 & 60.7 & 0.2 & 64.7 & 0.1 & 0.9 \\
WYT-8 & 2.7 & 0.7 & 42.2 & 0.3 & 45.9 & 0.1 & 0.9 \\
WYT-9 & 2.7 & 0.9 & 192.7 & 0.8 & 197.0 & 0.0 & 1.0 \\
WYT-10 & 12.8 & 15.6 & 45.1 & 1.7 & 75.2 & 0.6 & 0.6 \\
WYT-11 & 1.3 & 0.7 & 16.5 & 0.1 & 18.5 & 0.1 & 0.9 \\
WYT-12 & 2.2 & 0.7 & 11.7 & 0.1 & 14.7 & 0.3 & 0.8 \\
WYT-13 & 1.7 & 0.8 & 5.8 & 0.1 & 8.4 & 0.4 & 0.7 \\
WYT-14 & 1.6 & 0.3 & 16.2 & 0.1 & 18.2 & 0.1 & 0.9 \\
WYT-15 & 3.3 & 1.3 & 10.0 & 0.2 & 14.9 & 0.5 & 0.7 \\
WYT-16 & 1.3 & 7.1 & 24.4 & 0.2 & 33.0 & 0.3 & 0.7 \\
WYT-17 & 1.1 & 0.7 & 16.7 & 0.5 & 19.1 & 0.1 & 0.9 \\
WYT-18 & 1.8 & 0.4 & 17.1 & 0.3 & 19.6 & 0.1 & 0.9 \\
WYT-19 & 2.4 & 0.5 & 31.9 & 0.2 & 35.0 & 0.1 & 0.9 \\
WYT-20 & 1.6 & 0.5 & 42.4 & 0.1 & 44.6 & 0.0 & 1.0 \\
WYT-21 & 0.8 & 4.2 & 11.0 & 0.6 & 16.5 & 0.1 & 0.7 \\
\hline
\end{tabular}

$\mathrm{HCHs}$ pollution is mainly caused by the usage of $\mathrm{HCHs}$ and lindane. In China, after $\mathrm{HCHs}$ were banned, lindane has been extensively used in agricultural production; therefore, the components of $\mathrm{HCH}$ isomers have been used as indicators for environmental pollution (USEPA, 2010). The components of $\mathrm{HCHs}$ include $\alpha-\mathrm{HCH}$ $(55 \%-60 \%), \beta-\mathrm{HCH}(5 \%-14 \%), \gamma-\mathrm{HCH}(12 \%-16 \%), \delta-\mathrm{HCH}(6 \%-8 \%), \varepsilon-\mathrm{HCH}(2 \%-$ $9 \%)$, heptachlorocyclohexane (4\%), and octochlorocyclohexane $(0.6 \%)$. In lindane, the content of $\gamma-\mathrm{HCH}$ is $>90 \%$. Among the four $\mathrm{HCH}$ isomers, $\beta-\mathrm{HCH}$ is the easily absorbed by the soil but is the most difficult to be degraded by microorganisms in the soil. In the natural environment, $\alpha-\mathrm{HCH}$ and $\gamma-\mathrm{HCH}$ can be converted to $\beta-\mathrm{HCH}$. The average percentage of $\alpha-\mathrm{HCH}, \beta-\mathrm{HCH}, \gamma-\mathrm{HCH}$, and $\delta-\mathrm{HCH}$ detected in this study is $9 \%$, 
$26.33 \%, 11.90 \%$, and $3.6 \%$ of the average content of $\mathrm{HCH}$. Previously, studies have shown that the ratio of $\alpha-\mathrm{HCH} / \gamma-\mathrm{HCH}$ can be used to analyze the source of HCHs. In particular, when the ratio is $<1$, HCHs are mainly derived from the usage of lindane; when the ratio is near 0 , it indicates that lindane has been used recently (Cui et al., 2014); when the ratio of $\mathrm{HCH} / \gamma-\mathrm{HCH}$ falls between 3.44 and 5.00, the source of $\mathrm{HCHs}$ may be industrial production; and when the ratio exceeds 5.00, the HCHs may be the previous use of HCHs or biodegradation reactions (Cui et al., 2014). The results (Table $5)$ show that $33 \%$ of the sampling sites have a ratio that approaches $0(\alpha-\mathrm{HCH} / \gamma-\mathrm{HCH}$ is below 0.4 ) and $57 \%$ of the sampling sites have a ratio below 1(0.2-1.0), which indicates that lindane has been recently used in some areas around Wenyang Field. Most areas have no recent use of lindane.

Table 5. Proportions of HCHs for source identification in surface soil of the study area $\left(n g \cdot g^{-1}\right)$

\begin{tabular}{c|c|c|c|c|c|c|c|c|c|c|c}
\hline Samples & $\boldsymbol{\alpha}-\mathbf{H C H}$ & $\boldsymbol{\gamma}$-HCH & $\boldsymbol{\beta}-\mathbf{H C H}$ & $\boldsymbol{\delta}-\mathbf{H C H}$ & $\begin{array}{c}\boldsymbol{\alpha}-\mathbf{H C H} / \\
\boldsymbol{\gamma} \text {-HCH }\end{array}$ & $\mathbf{S a m p l e s}$ & $\boldsymbol{\alpha}-\mathbf{H C H}$ & $\boldsymbol{\gamma}-\mathbf{H C H}$ & $\boldsymbol{\beta}-\mathbf{H C H}$ & $\boldsymbol{\delta}-\mathbf{H C H}$ & $\begin{array}{c}\boldsymbol{\alpha}-\mathbf{H C H} / \\
\boldsymbol{\gamma}-\mathbf{H C H}\end{array}$ \\
\hline WYT-1 & 0.1 & 0.3 & 0.9 & 0.3 & $\mathbf{0 . 4}$ & WYT-12 & 0.1 & 0.4 & 1.7 & 0.6 & $\mathbf{0 . 3}$ \\
\hline WYT-2 & 0.6 & 0.5 & 1.1 & 0.1 & 1.1 & WYT-13 & 0.5 & 0.3 & 0.8 & 0.3 & 2.1 \\
\hline WYT-3 & 1.0 & 0.8 & 1.5 & 0.2 & 1.3 & WYT-14 & 0.4 & 0.7 & 1.7 & 0.1 & 0.6 \\
\hline WYT-4 & 0.2 & 0.8 & 1.1 & 0.1 & $\mathbf{0 . 2}$ & WYT-15 & 0.5 & 0.4 & 0.7 & 0.1 & 1.3 \\
\hline WYT-5 & 0.7 & 0.7 & 1.9 & 0.1 & 1.1 & WYT-16 & 0.6 & 2.2 & 1.2 & 0.1 & $\mathbf{0 . 3}$ \\
\hline WYT-6 & 0.6 & 1.3 & 1.3 & 0.1 & $\mathbf{0 . 4}$ & WYT-17 & 0.6 & 0.5 & 1.2 & 0.1 & 1.2 \\
\hline WYT-7 & 0.3 & 0.6 & 1.4 & 0.1 & $\mathbf{0 . 4}$ & WYT-18 & 0.6 & 0.4 & 1.0 & 0.6 & 1.7 \\
\hline WYT-8 & 0.7 & 1.1 & 1.9 & 0.1 & 0.6 & WYT-19 & 1.1 & 0.7 & 1.7 & 0.3 & 1.7 \\
\hline WYT-9 & 1.1 & 0.6 & 2.7 & 0.3 & 1.9 & WYT-20 & 0.4 & 0.4 & 1.0 & 0.1 & 1.0 \\
\hline WYT-10 & 1.7 & 3.4 & 8.8 & 1.4 & 0.5 & WYT-21 & 3.8 & 3.0 & 8.5 & 0.7 & 1.3 \\
\hline WYT-11 & 0.4 & 1.6 & 3.8 & 0.6 & $\mathbf{0 . 3}$ & & & & & & \\
\hline
\end{tabular}

\section{Conclusion}

Using gas chromatography, we detected the content of OCPs in 21 topsoil samples of Wenyang Field and analyzed the residue characteristics, health risks, ecological risks, and potential sources of OCPs. We obtained the following results. First, the major components of the 23 types of OC pollutants are DDTs $(6.76-222.76 \mathrm{ng} / \mathrm{g}$ ), endrin aldehyde, heptachlor epoxide, and HCHs (1.22-17.34 ng/g). Note that the detection rates of HCHs and DDTs in the topsoil of the study area can reach $100 \%$. As shown by the map of distribution of OCPs, the pollution level is high in Dawenkou Town and Wenyang Town, while the pollution is relatively low in Ciyao Town and Jiangji Town. Second, when analyzing the ecological risks of OCPs, it is revealed that OCPs pose high ecological risk to soil worms and birds but pose no obvious threats to mammals. Third, the analysis of health risks of OCPs shows that the carcinogenic risk associated with exposure to OCPs through different channels is below $10^{-6}$, which indicates that the carcinogenic risk of OCPs in the soil of Wenyang Field is small and negligible. Note that the value of non-carcinogenic risk (represented by hazard index) of each type of OCPs is far below 1, which indicates that the non-carcinogenic risk associated with exposure to OCPs in the soil of Wenyang Field is small and almost negligible. Fourth, the analysis of the sources of OCPs in the study area reveals that OCPs are mainly 
derived from residues of OCPs used previously and because of the recent use of lindane. To conclude, the development of biological agents that can clean the soil is important reduction in the usage of pesticides and fertilizers should be encouraged.

Follow-up research can be carried out, (1) A total of 21 samples were collected on the north and south of Dawen River this time, and the number of samples can be increased in the later. (2) Some organic pollutions, such as DDTs and HCHs, are hard to be degraded. In addition to the influence of natural and cultural environment, we can take samples and test in the same place several years later to see whether the pollution concentration will change significantly with time.(3) we can increase humanistic survey to know the changes of frequency, brand and efficacy of pesticides used by local farmers in recent years, Deeply understand the source of organic pollution.

Acknowledgements. This study was supported by National Natural Science Foundation of China (41402319), Supported by the Laboratory for Marine Geology, Qingdao National Laboratory for Marine Science and Technology( MGQNLM201802), Shandong Social Science Fund (18CLYJ55), Project of Shandong Natural Science Foundation (ZR2014DL002, ZR2013DL010), Scientific Research Program of Shandong Universities (J18KA197), and Taishan University Talent Research Fund (Y-01-2016001).

\section{REFERENCES}

[1] An, Q., Dong, Y., Wang, H., Ge, C. (2005): OCP residues in soil in Nanjing and distribution characteristics. - Acta Scientiae Circumstantiae 25(4): 470-474.

[2] Chen, F., Wang, J., You, Y., Wang, C. (2011): Characteristics of OCP pollution in soil of vegetable-growing bases in Fuzhou and risk assessment. - Chinese Journal of Tropical Crops 6: 1185-1189.

[3] Chen, L., Feng, Q., He, Q., Huang, Y., Zhang, Y., Jiang, G., Zhao, W., Gao, B., Lin, K., $\mathrm{Xu}$, Z. (2017): Sources, atmospheric transport and deposition mechanism of organochlorine pesticides in soils of the Tibetan Plateau. - Science of the Total Environment 577: 405-412.

[4] Cheng, H., Ding, P., Zhang, M. (2018): Characteristics of pollution of OCP residues in farmland soil in Henan. - Jiangsu Agricultural Sciences 46(14): 247-252.

[5] Cheng, T. (2014): Spatial Distribution of OCP in Soil of Agricultural Areas in Suburban Shenyang and Risk Assessment. - Shenyang University, Shenyang.

[6] Cui, J., Du, J. Z., Ma, H., Wang, X. (2012): Organic pollution assessment of soil in suburban Shenyang. - Acta Ecologica Sinica 34(24): 7874-7882.

[7] Cui, J., Wang, X., Du, J., Yang, Z., Yue, M., Li, X., Ma, H. (2014): Characteristics of OCP residues of surface soil in suburban Shenyang and risk assessment. - Geology in China 41(5): 1705-1715.

[8] Dai, J., Zhang, J., Yu, C., Pang, X. (2012): Research on OCP residues and sources in soil of Yantai of Shandong. - Earth and Environment 40(1): 50-56.

[9] Jiang, Y., Wang, X., Sun, Y., Sheng, G., Fu, J. (2010): Research on OCP residues in soil of urban areas of Shanghai. - Environmental Science 31(2): 409-414.

[10] Jongbloed, R. H., Traas, T. P., Luttik, R. (1996): A probabilistic model for deriving soil quality criteria based on secondary poisoning of top predators: calculations for DDT and Cadmium. - Ecotoxicology and Environmental Safety 34(3): 279-306.

[11] Lin, T., Qin, Y., Zhang, L., Zheng, B., Li, Y., Guo, Z. (2011): Distribution, sources and risk assessment of OCP and PCB residues in sediments of Dahuofang Reservoir in Niaoning. - Environmental Science 23(11): 3294-3299. 
[12] Liu, H., Liu, M., Wu, D., Zheng, J., Zhan, C., Zhang, J., Yao, R. (2013): Characteristics of OCP residues in central and northern farmland in Jilin. - Journal of Jilin University (Science Edition) 51(6): 1187-1192.

[13] Liu, H., Liu, M., Wu, D., Zheng, J., Zhan, C., Zhang, J., Yao, R. (2017): Characteristics of OCP pollution in eastern Hubei province and health risk assessment. - Journal of Hubei Polytechnic University 33(3): 19-23.

[14] Long, E. R., Macdonald, D. D., Smith, S. L., Calder, F. D. (1995): Incidence of adverse biological effects within ranges of chemical concentrations in marine estuarine sediment. - Environ. Manag. 19(1): 81-97.

[15] Ma, J., Qiu, Y., Zhou, H., Zhu, T. (2015): Research on spatial distribution of OCP residues in typical areas of Pearl River Delta based on multivariate geostatistics and GIS a case study of Huizhou. - Acta Pedologica Sinica 47(3): 339-450.

[16] Meng, P., Li, R., He, Y., Zhang, A., Zhang, L., Jin, M., Liang, Q., Zhang, L. (2017a): Characteristics of pollution of OCP in soil of pastoral areas of Baotou and health risk assessment. - Modern Preventive Medicine 44(1): 32-36, 56.

[17] Meng, P., Li, R., He, Y., Zhang, A., Zhang, L., Jin, M., Liang, Q., Zhang, L. (2017b): Distribution characteristics of OCP in soil of pastoral areas in Inner Mongolia and health risk assessment. - Journal of Agro-Environment Science 36(3): 539-546.

[18] Niu, L., Xu, C., Yao, Y., Liu, K., Yang, F., Tang, M., Liu, W. (2013): Status, influences and risk assessment of hexachlorocyclohexanes in agricultural soils across China. Environmental Science \& Technology 47: 12140-12147.

[19] Pang, X., Zhang, F., Wang, H., Hu, X., Zeng, X. (2009): OCP residues and distribution characteristics in soil in southwestern Shangdong province. - Geological Bulletin of China 28(5): 667-670.

[20] Shi, L., Sun, Y., Lv, A., Cai, X., Shen, X., Shen, J. (2016): Distribution characteristics of 22 OCP in soil in Yangtze River Delta. - Rock and Mineral Analysis 35(1): 75-81.

[21] United States Environmental Protection Agency (USEPA) (1996): Soil Screening Guidance: Technical Background Document. - EPA/540/R-95/128. Office of Solid Waste and Emergency Response, Washington, DC.

[22] United States Environmental Protection Agency (USEPA) (1997): Exposure Factors Handbook. - EPA/600/P-95/002F; Environmental Protection Agency, Office of Research and Development, Washington, DC.

[23] United States Environmental Protection Agency (USEPA) (2002): Supplemental Guidance for Developing Soil Screening Levels for Superfund Sites. - OSWER 9355.424, Washington, DC.

[24] United States Environmental Protection Agency (USEPA) (2010): Mid Atlantic Risk Assessment. - Regional Screening Level (RSL) Summary Table, Washington, DC.

[25] Wu, J. (2012): Classification and Evaluation of Buffering and Filtering Functions of Regional Soil - A Case Study of Suburban Areas of Zhengzhou. - China University of Geosciences, Beijing, pp. 11-13.

[26] Xiao, P. (2017): Research advances on OCP pollution in soil of Jilin province. Heilongjiang Science and Technology Information 17.

[27] Zhang, H., Gao, R., Jiang, S., Huang Y (2006): Spatial distribution of OCP residues in farmland soil in Beijing. - Scientia Agricultura Sinica 39(7): 1403-1410.

[28] Zhang, J. (2016): OCP Residues in Soil-Vegetable System in Suburban Areas of Jilin and Risk Assessment. - University of Chinese Academy of Sciences, Beijing, pp. 63-77.

[29] Zhao, X., Lu, H., Luo, H., Li, R., Zhu, Y. (2006): Research on OCP residues in farmland in Cixi City. - Journal of Ningbo University (Natural Science \& Engineering) 19(1): 98100.

[30] Zhu, Y., You, Z., Shen, T., Zhong, H., Chai, L. (2012): OCP and PCB residues in sediments in shellfish culturing areas of intertidal zones in Ningbo and ecological risk assessment. - Chinese Journal of Applied Ecology 23(6): 1689-1694. 